\title{
Simultaneous Optimization of Pump Configuration and Pipe Network in Multi-source Water Injection System
}

\author{
Jianjun YANG ${ }^{{ }^{*}}$, Tangfu Wu $\mathrm{W}^{\mathrm{b}}$ and Zhihao $\mathrm{Liu}^{\mathrm{c}}$ \\ School of Mechanical Engineering, Qingdao University of Technology, Qingdao, 266520, China \\ aemail: yjjdem@163.com, bemail: 778694626@qq.com, email: 1143273559@qq.com
}

Keywords: Water Injection System, Pump Configuration, Pipe Network Optimization, Simultaneous Optimization, Improved Dual Coding Genetic Algorithm

Abstract. The simultaneous optimization of pump configuration and pipe network can greatly improve the system operating efficiency. Aiming at the new-built and multi-source water injection system, the optimal mathematical model was established, in which the operating energy consumption of pumps and the investment of pumps and pipe networks are served as objective function, the type, number and operation parameters of pumps, and the connection status, diameter of pipeline are taken as design variables. The improved dual coding genetic algorithm was designed to solve the problem. In the algorithm, the dual generalized chromosome coding method, the determination method of pump type, the generation method of looped pipe network, and the crossover and mutation methods were designed. The example shows the efficiency of the optimization method.

\section{Introduction}

In the production process of oilfield, water injection system is an important power consumer. The water injection system is a multi-source and looped pipe network system in the larger oil production plant. The pump and pipe network efficiency are important components of system running efficiency, the optimization of pumps and pipe networks are very important to enhance the efficiency and reduce the cost, and the pump operating status and pipe network status are influenced each other. But the existing researches only optimize one kind of problems in pump [1,2] and pipe network [3,4] optimization. In order to optimize the new-built system completely, the simultaneous optimization of pump and pipe network is necessary.

\section{The Optimal Mathematical Model}

In the optimization objective function, the energy consumption of pumps, the investment of pumps and pipe networks are considered, so the type, number and operation parameters of pumps, and the connection status, diameter of pipelines are taken as design variables.

$$
\min f^{\prime}=\sum_{i=1}^{N_{\mathrm{p}}}\left(C_{\mathrm{m} i}+\omega_{1} T C_{\mathrm{d}} \gamma \frac{H_{i} Q_{i}}{\eta_{\mathrm{p} i} \eta_{\mathrm{m} i}}\right)+\omega_{2} \sum_{j=1}^{N_{\mathrm{L}}} \xi_{j} L_{j} C_{\mathrm{l} j}\left(D_{j}\right)
$$

where $N_{\mathrm{p}}$ is the total number of the running pumps, $C_{\mathrm{m} i}$ is the pump and drive motor investment cost of pump $i$, correspond to the pump type, $\omega_{1}$ is the adjustment coefficient between the operation cost and investment cost, $T$ is the investment recovery period, $C_{\mathrm{d}}$ is unit price of power, $\gamma$ is unit conversion factor, $H_{i}, Q_{i}, \eta_{\mathrm{p} i}, \eta_{\mathrm{m} i}$ are the head, flow rate, efficiency and drive motor efficiency of pump $i$ respectively, and $\eta_{\mathrm{m} i}$ can be regard as constant[5], $\omega_{2}$ is the adjustment coefficient between the two parts of cost, $N_{\mathrm{L}}$ is the total number of pipelines in preliminary connection graph of pipe network, $\xi_{j}$ is connection status of the pipeline $j$, the value is 1 or 0 corresponding connection or disconnection, $L_{j}$ is the length of pipeline $j, C_{1 j}$ is the unit cost of pipeline $j$, correspond to the pipe diameter.

At the same time, the following constraints should be satisfied. 
The first constraint is water balance constraint. The sum of pump flow rates is equal to the water demand of the system, and is a constant.

$$
\sum_{i=1}^{N_{\mathrm{p}}} Q_{i}=Q_{\mathrm{All}}
$$

where $Q_{\text {All }}$ is the water demand of the system.

The second set of constraints is hydraulic equilibrium constraint [2]. The constraint is satisfied automatically in the simulation process. But the node pressure values are relative values that relative to the reference node, all the node pressure values increase or decrease a fixed value, the equilibrium equations are still met.

The third set of constraints is node pressure constraint. The pressure value of each water position should be not less than the minimum working pressure.

$$
p_{k} \geq p_{k \min }
$$

where $p_{k}, p_{k \min }$ are the calculation pressure value and minimum working pressure of water position $k$ respectively.

The fourth set of constraints is pump flow rate constraint. The flow rate of each type of pump should be limited within a range to ensure the efficient operation.

$$
Q_{i \min } \leq Q_{i} \leq Q_{\text {imax }}
$$

where $Q_{i \min }, Q_{i \max }$ are minimum and maximum flow rate of pump $i$ respectively, and the values are related to the pump type.

The fifth set of constraint is pump working pressure constraint. In order to guarantee normal operation of pump, the outlet pressure of pump should be greater than the node pressure of water injection station, and the pressure difference is called pump-pipe pressure difference. After simulation calculation of the system, all the node pressure values are adjusted uniformly, then each pump outlet pressure can be greater than the node pressure of station, and In order to get the optimal solution, the pump-pipe pressure difference should be kept as small as possible. Because there is friction loss, the minimum value of pump-pipe pressure difference is set to $0.05 \mathrm{MPa}$ in this paper. So the pump working pressure constraint is satisfied in the simulation process.

The sixth constraint is looped pipe network constraint. All the pipelines in connection status should be a looped pipe network.

The seventh set of constraints is pipe diameter constraint. The diameter of each pipe must belong to the set of optional standard diameters.

The final constraint is water supply constraint. All the water positions can be supplied with water.

Through the designs of the improved algorithm, only the node pressure constraint can't be satisfied directly, so the penalty function method is adopted, and the final objective function is formed, that is

$$
f=f^{\prime}+M \sum_{k=1}^{N_{\mathrm{n}}} \max \left(0, p_{k \min }-p_{k}\right)
$$

where $M$ is the penalty parameter, $N_{\mathrm{n}}$ is the total number of water positions.

\section{The Main Solution Steps of the Improved Dual Coding Genetic Algorithm}

Determining the Optimization Variables and Pump Type. Based on the objective function, the number, type, head, flow rate, efficiency of running pumps, and the connection status, diameter of pipelines all need to be optimized. But when the pump type and flow rate are given, the head and efficiency of pump can be obtained through corresponding characteristic curves. In order to improve the search efficiency of the algorithm, when a flow rate is given, the pump type can't be determined randomly, it can be determined according to the following methods: 1 ) if the flow rate only in one flow rate constraint range of pump type, then select the pump type. 2) if the flow rate belongs to several flow rate constraint ranges of pump type, we can compute the function value according to the first part of formula 1 and pressure penalty term in formula 5, 
select the pump type corresponding to the minimum value. Through the above analysis, only the number, flow rate of the pumps, and the connection status, diameter of pipelines are selected as direct optimization variables finally.

Coding Design. The flow rate is a real number, so the real number coding is adopted, and the length of coding is equal to the number of pumps. The connection status of pipeline can be expressed by 0 or 1 , so binary coding is adopted. The diameter of pipeline is discrete variable, integer coding is adopted. If there are $N_{\mathrm{sl}}$ kinds of diameter, an integer between 1 and $N_{\mathrm{sl}}$ corresponds to one diameter.

The flow rates are generated randomly one by one, when the sum of flow rates is close to the $Q_{\text {All }}$, the flow rates are adjusted, until the water balance constraint is satisfied, and ensure that each flow rate belongs to at least one flow rate constraint range of pump.

Based on the generalized chromosome coding method[6], dual generalized chromosome coding is put forward. Optimal variables about pump on the left side of chromosome, and optimal variables about pipeline on the right side. On the left side, in the first line, the real number coding is adopted to express the flow rate of pump, in the second line, the integer coding is adopted to express the water injection station that the pump belongs to, the length of coding is equal to $N_{\mathrm{p}}$. On the right side, in the first line, the binary coding is adopted to express the connection status of pipeline, in the second line, the integer coding is adopted to express the diameter of pipeline, the length of coding is equal to $N_{\mathrm{L}}$. An example of coding is shown in Fig.1.

$\begin{array}{cccc:cccccc}276.5 & 308.2 & 387.4 & 401.6 & 1 & 1 & 0 & 0 & 1 & 1 \\ 3 & 2 & 1 & 4 & 2 & 1 & 4 & 2 & 3 & 2\end{array}$

Fig. 1 The diagram of dual generalized chromosome coding

For the binary coding of pipeline, in order to avoid the infeasible solutions in the initial population, the generating method is designed based on the concept of cycle in the graph theory. The generating method of looped pipe network can be divided into two steps. Firstly, all binary coding are equal to 1, i.e. all the pipelines are in connection status, the preliminary connection graph is formed. Secondly, randomly select partial cycles in the preliminary connection graph, then judge whether disconnected pipelines exist in those cycles one by one, if not, disconnect any pipeline in the loop randomly, that is turn the gene value to 0 , and ensure at least one cycle exist in the graph.

Crossover operation. One method is select randomly from the following methods. 1) Real number coding crossover of pump. The arithmetic crossover method is adopted, and the water balance constraint is satisfied automatically in the offspring. But the pump type could be about to change, judgment and adjustment are needed. 2) Integer coding crossover of pump. Single point crossover method is adopted, only the stations pump belongs to are changed, all the constraints are not affected. 3) Binary coding crossover of pipeline. Single point crossover or coding interchange is adopted randomly. If the single point crossover is adopted, when selecting cross point, we should compute the number of connected pipelines in two parent chromosomes, and ensure that the relation between pipeline quantity in connection status and the node quantity satisfied basic requirement of looped network. If the coding interchange is adopted, the coding is still feasible solution. 4) Integer coding crossover of pipeline. The arithmetic crossover method is adopted, and the decimal should be rounded off, the pipe diameter constraint is satisfied automatically.

Mutation Operation. Four kinds of mutation methods are designed. 1) Real number coding mutation of pump. In order to satisfy the water balance constraint, two genes are selected randomly, one gene vale is increased or decreased a small value, and another gene value is decreased or increased the same value. The flow rates are changed, and the pump types need to be determined again. 2) Integer coding mutation of pump. One gene value is changed randomly, or two gene values are interchanged. 3) Binary coding mutation of pipeline. One gene that the value is 0 is selected, and changes the gene value into 1.4) Integer coding mutation of pipeline. One gene vale is increased or decreased a small integer, and in the range of 1 to $N_{\mathrm{L}}$. 


\section{Optimization Example}

A water injection system is optimized, there are 2 water injection stations in the system, and the water demand of the system is $187 \mathrm{~L} / \mathrm{s}$. In the current operation states of the system, there are two running pumps, pump types are D300-150×11 and D400-150×11, the total investment of pumps and drive motors is 1.125 million Yuan, the total power is $4057 \mathrm{~kW}$, and the total investment of pipe network is 11.554 million Yuan. In the optimization process, the adjustment coefficient $\omega_{1}=0.02, \omega_{2}=0.5$, the investment recovery period $T=3$ Year, the unit price of power $C_{\mathrm{d}}=0.6 \mathrm{Yuan} / \mathrm{kW} \cdot \mathrm{h}$. After the simultaneous optimization, pump type are D280-160A $\times 11$ and D300-150×11, the total investment of pumps and drive motors is 1.023 million Yuan, the total power is $3860 \mathrm{~kW}$, and the total investment of pipe network is 10.031 million Yuan. The optimization effect is obvious.

\section{Conclusion}

In order to reduce the investment cost and enhance the operation efficiency, the simultaneous optimization of pump configuration and pipe network is necessary. The optimal mathematical model is established, the investment of pumps and pipe networks, and the energy consumption of pumps are considered, the number, flow rate of the pumps, and the connection status, diameter of pipelines are selected as direct optimization variables. In the improved dual coding genetic algorithm, the dual generalized chromosome coding method is adopted, and the methods of coding, crossover and mutation are designed, some constraints are satisfied automatically.

\section{Acknowledgement}

In this paper, the research was sponsored by the Project of Shandong Province Higher Educational Science and Technology Program (Project No. J13LB13).

\section{References}

[1] Broad D R, Maier H R, Dandy G C, Asce M. Optimal operation of complex water distribution systems using metamodels. Journal of Water Resources Planning and Management, 2010, 136(4):433-443.

[2] Yang J J, Liu Y, Wei L X, Zhan H. Dual coding hybrid genetic algorithm for optimal schedule of pumping stations in multi-sources water injection system. Acta Automatica Sinica, 2006,32(1):154-160.

[3] Zheng F F, Simpson A R, Zecchin A C. Dynamically expanding choice-table approach to genetic algorithm optimization of water distribution systems. Journal of Water Resources Planning and Management, 2011,137(6):547-551.

[4] Mohan S, Babu K S. Optimal water distribution network design with honey-bee mating optimization. Journal of Computing in Civil Engineering, 2010,24(1):117-126.

[5] Cheng Jilin, Zhang Lihua, Zhang Rentian, et al. Optimal methodology of Single-un it variable speed operation in pumping station. Transactions of the Chinese Society for Agricultural Machinery, 2010,41(3):72-76.

[6] Yang J H, Wu C G, Lee H P, et al. Solving traveling salesman problems using generalized chromosome genetic algorithm. Progress in Natural Science, 2008,18(7):887-892. 